\title{
Bayesian Inference for Exponential Lifetime Models Based on Type-II Hybrid Censoring
}

\author{
Husam Awni Bayoud \\ College of Sciences and Humanities, Fahad Bin Sultan University, Tabuk 71454, Saudi Arabia \\ E-mail: hbayoud@fbsu.edu.sa \\ Received 15 March 2013 \\ Accepted 20 April 2014
}

This article deals with the problem of estimating the parameters of the two-parameter exponential lifetime distribution based on Type-II hybrid censored samples from the Bayesian viewpoint. The scale and location parameters are assumed to have exponential and uniform priors respectively. Bayes point estimates and credible intervals for the unknown parameters are derived under the assumption of the squared error loss function. A lifetime real dataset is analyzed to motivate and to show the performance of the proposed Bayes estimates based on Type-II hybrid censoring scheme. Various Simulation studies are also provided in this paper to compare the proposed Bayes estimates with the existing classical estimates.

Keywords: Bayes Estimation; Credible Interval; Exponential Distribution; Hybrid Censored Samples; Kolmogorov-Smirnov Test; Squared Error Loss Function.

2000 Mathematics Subject Classification: 62F15, 62N01.

\section{Introduction}

Let $X_{1}, X_{2}, \ldots, X_{n}$ be a random sample of size $n$ from a two-parameter exponential distribution $E(\theta, \lambda)$ with scale parameter $\theta$ (i.e. with mean lifetime of $1 / \theta$ ) and location parameter (guarantee lifetime) $\lambda$. The parameters $\theta$ and $\lambda$ are independent. The probability density function (p.d.f) of $X$ at $x$ is:

$$
f(x \mid \theta, \lambda)=\theta e^{-\theta(x-\lambda)} \quad ; \theta>0,0 \leq \lambda \leq x
$$

This distribution plays an important role in survival and reliability analysis (see [9] for instance). If the lifetime of an electrical component follows a two-parameter exponential distribution, 
then the mean lifetime of this component is $1 / \theta$ and the guarantee lifetime of this component is $\lambda$. It is very important in this situation to estimate the component's lifetime mean and guarantee lifetime.

In life testing experiments, it often happens that the experiment is censored in the sense that the experimenter may not be in a position to observe the life times of all items put on test because of time limitations and other restrictions on the data collection. The two most common censoring schemes are Type-I and Type-II censoring schemes. In Type-I censoring scheme, the experiment continue up to a preselected fixed time $T$ but the number of failures is random, whereas in Type-II censoring scheme, the experimental time is random but the number of failures is fixed, $k$. A mixture of Type-I and Type-II censoring schemes is known as the hybrid censoring scheme and it can be described as follows. Suppose a total of $n$ units is placed on a life testing experiment, and the lifetimes of the sample units are independent and identically distributed (i.i.d.) random variables. Let the ordered lifetimes of these items are denoted by $x_{(1)}<x_{(2)}<\ldots<x_{(k)}<\ldots<x_{(n)}$ respectively. The test is terminated when a preselected number of failures $k$ out of $n$ items are failed, or when a preselected time $T$ on test has been reached, i.e., the test is terminated at the time point $T_{*}=\min \left(T, x_{(k)}\right)$, where $x_{(k)}$ is the $\mathrm{k}^{\text {th }}$ ordered lifetime. This censoring scheme was introduced firstly by Epstein [4], and it is popularly known as Type-I hybrid censoring scheme. It has been used quite extensively in reliability acceptance test in MIL-STD-781-C [8]. The censoring scheme in which the experiment is terminated at the time point $T^{*}=\max \left(T, x_{(k)}\right)$ is known as Type-II hybrid censoring scheme, this censoring scheme was introduced by Childs et al. [1]. This censoring scheme guaranteeing that at least $k$ failures are observed at the end of the experiment. Whereas, the number of observed failures based on Type-I hybrid censoring scheme may equal zero if the preselected $T$ is less than $x_{(1)}$. So, in this case the inferential results are obtained under the condition that the number of observed failures is at least one, and moreover, there may be very few failures at the termination point of the experiment. In that case the efficiency of the estimator(s) may be very low.

Classical literature for estimation the parameters of a two parameter exponential distribution based on hybrid censored samples includes Epstein [4], Lawless (1977), Childs et al. [1], Childs et al. [2] and Ganguly et. al. [3]. This estimation problem has also been considered by several authors in the literature from the Bayesian point of view. Draper and Guttmann [10] considered the problem of estimating the one parameter exponential distribution from the Bayesian point of view based on TypeII hybrid censored sample. Singh and Prasad [11] and Prasad and Singh [5] proposed empirical Bayes estimate for the location parameter $\lambda$ under the situation that the mean lifetime parameter is known based on complete sample. Recently, Bayoud [7] introduced Bayes estimates for the parameters of the Two-parameter exponential distribution based on Type-I censored samples.

In this paper, Bayes estimates for the scale and location parameters of a two exponential distribution are derived based on a Type-II hybrid censored sample. The scale parameter is assumed to follow exponential distribution with hyper parameter $A$, and the location parameter is assumed to follow uniform distribution from zero to $B$. The squared error function is assumed. Suggestions for choosing the hyper parameters $A$ and $B$ are provided. 
The rest of this paper is organized as follows: Section 2 describes the probability models that are needed in this work. Bayes and maximum likelihood estimates for the scale and location parameters are derived in Section 3 based on complete and Type-II hybrid censored samples. An illustrative example is provided in Section 4. Simulation studies are presented in Section 5. Finally, the main conclusions are included in Section 6.

\section{The Models}

\subsection{Complete Sample}

Let $X_{1}, X_{2}, \ldots, X_{n} \sim E(\theta, \lambda)$. The likelihood function of the complete sample $X_{1}, X_{2}, \ldots, X_{n}$ given $\theta$ and $\lambda$ is given by:

$$
L\left(x_{1}, x_{2}, \ldots, x_{n} \mid \theta, \lambda\right)=\theta^{n} e^{-\theta \sum_{i=1}^{n}\left(x_{i}-\lambda\right)}
$$

where $X_{i} \geq \lambda \geq 0 ; i=1,2, \ldots, n$ and $\theta>0$.

The parameter $\theta$ is assumed to follow exponential distribution with p.d.f given by:

$$
g(\theta)=A e^{-A \theta} ; A>0
$$

where the hyper parameter $A$ is a preselected positive real number that is chosen to reflect our beliefs about the expected value of $1 / \theta$, because the expected value of $\theta$ equals $1 / A$. The hyper parameter $A$ can be easily assumed to equal one over the available sample's mean.

The parameter $\lambda$ is assumed to follow a uniform distribution with p.d.f given by:

$$
p(\lambda)=\frac{1}{B} ; 0<\lambda \leq B \leq x_{(1)}
$$

where the hyper parameter $B$ is a preselected positive real number that is chosen to reflect our beliefs about the lower bound of the x's , which can be easily assumed to equal the minimum observed value, $x_{(1)}$.

Based on the complete sample $\left\{x_{1}, x_{2}, \ldots, x_{n}\right\}$ the joint posterior p.d.f of $\theta$ and $\lambda$ is given by:

$$
h_{C}\left(\theta, \lambda \mid x_{1}, x_{2}, \ldots, x_{n}\right)=\frac{L\left(x_{1}, x_{2}, \ldots, x_{n} \mid \lambda, \theta\right) g(\theta) p(\lambda)}{\int_{0}^{B} \int_{0}^{\infty} L\left(x_{1}, x_{2}, \ldots, x_{n} \mid \lambda, \theta\right) g(\theta) p(\lambda) d \theta d \lambda}=\frac{n \theta^{n} e^{-\theta\left[A+\sum_{i=1}^{n}\left(x_{i}-\lambda\right)\right]}}{C \Gamma(n)}
$$


where $C=\frac{1}{D^{n}}-\frac{1}{E^{n}} \neq 0$ in which $E=A+\sum_{i=1}^{n} x_{i}, D=E-n B, \theta>0$ and $0 \leq \lambda \leq B \leq x_{(1)}$.

The marginal posterior p.d.fs of $\theta$ and $\lambda$ given $\left\{x_{1}, x_{2}, \ldots, x_{n}\right\}$ are respectively given by:

$$
\begin{aligned}
h_{\theta, C}\left(\theta \mid x_{1}, x_{2}, \ldots, x_{n}\right) & =\int_{0}^{B} h\left(\theta, \lambda \mid x_{1}, x_{2}, \ldots, x_{n}\right) d \lambda \\
& =\frac{\theta^{n-1}}{C \Gamma(n)}\left(e^{-D \theta}-e^{-E \theta}\right) \quad ; \theta>0
\end{aligned}
$$

and

$$
\begin{aligned}
h_{\lambda, C}\left(\lambda \mid x_{1}, x_{2}, \ldots, x_{n}\right) & =\int_{0}^{\infty} h\left(\theta, \lambda \mid x_{1}, x_{2}, \ldots, x_{n}\right) d \theta \\
& =\frac{n^{2}}{C} \frac{1}{\left(A+\sum_{i=1}^{n}\left(x_{i}-\lambda\right)\right)^{n+1}} \quad ; 0<\lambda \leq B \leq x_{(1)}
\end{aligned}
$$

\subsection{Type II Hybrid Censored Sample}

In Type-II hybrid censored samples, the test is terminated when $k$, a preselected number, out of $n$ items are failed, or when a preselected time $T$ on test has been reached.

Let $r$ be the number of units failed before the time $T$, then the likelihood of the observed data under the Type-II hybrid censoring scheme is given by:

$$
L_{H}(\text { data } \mid \lambda, \theta)= \begin{cases}\frac{n !}{(n-r) !} \theta^{r} e^{-\theta\left[\sum_{i=1}^{r} x_{(i)}+(n-r) T-n \lambda\right]} & \text { if } x_{(k)}<T \\ \frac{n !}{(n-k) !} \theta^{k} e^{-\theta\left[\sum_{i=1}^{k} x_{(i)}+(n-k) x_{(k)}-n \lambda\right]} & \text { if } x_{(k)}>T\end{cases}
$$

The joint posterior p.d.f of $\theta$ and $\lambda$ based on the Type-II hybrid censored sample is given by: 


$$
h_{H}(\theta, \lambda \mid \text { data })=\frac{L_{H}(\text { data } \mid \lambda, \theta) g(\theta) p(\lambda)}{\int_{0}^{B} \int_{0}^{\infty} L_{H}(\text { data } \mid \lambda, \theta) g(\theta) p(\lambda) d \theta d \lambda}
$$

Therefore

$$
h_{H}(\theta, \lambda \mid \text { data })= \begin{cases}\frac{n}{\Gamma(r)} \frac{\theta^{r} e^{-\theta\left[E_{1}-n \lambda\right]}}{C_{1}} & \text { if } x_{(k)}<T \\ \frac{n}{\Gamma(k)} \frac{\theta^{k} e^{-\theta\left[E_{2}-n \lambda\right]}}{C_{2}} & \text { if } x_{(k)}>T\end{cases}
$$

where $\theta>0,0 \leq \lambda \leq B, C_{1}=\frac{1}{D_{1}^{r}}-\frac{1}{E_{1}^{r}}$ in which $E_{1}=\sum_{i=1}^{r} x_{(i)}+(n-r) T+A, D_{1}=E_{1}-n B$, and $C_{2}=\frac{1}{D_{2}^{k}}-\frac{1}{E_{2}^{k}}$ in which $E_{2}=\sum_{i=1}^{k} x_{(i)}+(n-k) x_{(k)}+A, D_{2}=E_{2}-n B$.

The marginal posterior p.d.fs of $\theta$ and $\lambda$ given Type-II hybrid censored data are respectively given by:

$$
\begin{aligned}
h_{\theta, H}(\theta \mid \text { data }) & =\int_{0}^{B} h_{H}(\theta, \lambda \mid \text { data }) d \lambda \\
& =\left\{\begin{array}{ll}
\frac{\theta^{r-1}\left(e^{-D_{1} \theta}-e^{-E_{1} \theta}\right)}{C_{1} \Gamma(r)} & \text { if } x_{(k)}<T \\
\frac{\theta^{k-1}\left(e^{-D_{2} \theta}-e^{-E_{2} \theta}\right)}{C_{2} \Gamma(k)} & \text { if } x_{(k)}>T
\end{array} ; \theta>0\right.
\end{aligned}
$$

and

$$
\begin{aligned}
h_{\lambda, H}(\lambda \mid \text { data }) & =\int_{0}^{\infty} h_{H}(\theta, \lambda \mid \text { data }) d \theta \\
& =\left\{\begin{array}{ll}
\frac{n r}{C_{1}} \frac{1}{\left[\sum_{i=1}^{r} x_{(i)}+(n-r) T-n \lambda+A\right]^{r+1}} & \text { if } x_{(k)}<T \\
\frac{n k}{C_{2}} \frac{1}{\left[\sum_{i=1}^{k} x_{(i)}+(n-k) x_{(k)}-n \lambda+A\right]^{k+1}} & \text { if } \quad x_{(k)}>T
\end{array} ; 0<\lambda \leq B \leq x_{(1)}\right.
\end{aligned}
$$




\section{Classical and Bayesian Estimation}

In this section, Classical and Bayesian estimation for $\theta$ and $\lambda$ are proposed using the complete and Type-II hybrid censored samples separately. The squared error loss is assumed to construct the Bayes estimates.

\subsection{Based on Complete Sample}

In the case of complete sample, the Bayes point estimate $\hat{\theta}_{B, C}$ of $\theta$ under the squared error loss is the mean of the marginal posterior p.d.f of $\theta$, which is given by:

$$
\begin{aligned}
\hat{\theta}_{B, C}=E_{h_{\theta, C}}(\theta) & =\int_{0}^{\infty} \theta h_{\theta, C}\left(\theta \mid x_{1}, x_{2}, \ldots, x_{n}\right) d \theta \\
& =\frac{n}{C}\left(\frac{1}{D^{n+1}}-\frac{1}{E^{n+1}}\right)
\end{aligned}
$$

Bayes point estimate $\hat{\lambda}_{B, C}$ of $\lambda$ under the squared error loss is the mean of the marginal posterior p.d.f of $\lambda$, which is given by:

$$
\begin{aligned}
\hat{\lambda}_{B, C}=E_{h_{\lambda, C}}(\lambda) & =\int_{0}^{B} \lambda h_{\lambda, C}\left(\lambda \mid x_{1}, x_{2}, \ldots, x_{n}\right) d \lambda \\
& =\frac{1}{C}\left(\frac{B}{D^{n}}+\frac{1}{n(1-n)}\left(\frac{1}{D^{n-1}}-\frac{1}{E^{n-1}}\right)\right)
\end{aligned}
$$

provided that $n>1$.

The MLE of $\theta$ and $\lambda$ from the complete sample are respectively:

$$
\hat{\theta}_{M L E, C}=\frac{n}{\sum_{i=1}^{n}\left(x_{i}-x_{(1)}\right)}, \quad \text { and } \quad \hat{\lambda}_{M L E, C}=x_{(1)}
$$

\subsection{Based on Type II Hybrid Censored Sample}

In the case of Type-II hybrid censored sample, the Bayes point estimate $\hat{\theta}_{B, H}$ of $\theta$ under the squared error loss is the mean of the marginal posterior p.d.f of $\theta$, which is given by:

$$
\hat{\theta}_{B, H}=E_{h_{\theta, H}}(\theta)=\int_{0}^{\infty} \theta h_{\theta, H}(\theta \mid \text { data }) d \theta
$$




$$
= \begin{cases}\frac{r}{C_{1}}\left(\frac{1}{D_{1}^{r+1}}-\frac{1}{E_{1}^{r+1}}\right) & \text { if } x_{(k)}<T \\ \frac{k}{C_{2}}\left(\frac{1}{D_{2}^{k+1}}-\frac{1}{E_{2}^{k+1}}\right) & \text { if } x_{(k)}>T\end{cases}
$$

The Bayes point estimate $\hat{\lambda}_{B, H}$ of $\lambda$ under the squared error loss is the mean of the marginal posterior p.d.f of $\lambda$, which is given by:

$$
\begin{aligned}
\hat{\lambda}_{B, H}=E_{h_{\lambda, H}}(\lambda) & =\int_{0}^{B} \lambda h_{\lambda, H}(\lambda \mid \text { data }) d \lambda \\
& =\left\{\begin{array}{l}
\frac{1}{C_{1}}\left(\frac{B}{D_{1}^{r}}+\frac{1}{n(1-r)}\left(\frac{1}{D_{1}^{r-1}}-\frac{1}{E_{1}^{r-1}}\right)\right) \text { if } x_{(k)}<T \\
\frac{1}{C_{2}}\left(\frac{B}{D_{2}^{k}}+\frac{1}{n(1-k)}\left(\frac{1}{D_{2}^{k-1}}-\frac{1}{E_{2}^{k-1}}\right)\right) \text { if } x_{(k)}>T
\end{array}\right.
\end{aligned}
$$

If $r=0$, then $x_{(k)}>T$. In this case if $k=1$ then $\hat{\lambda}_{B, H}$ does not exist. So, if $r=0$ then $\hat{\lambda}_{B, H}$ exists only if $k>1$. If $x_{(k)}<T$, then $r>k \geq 1$, therefore $\hat{\lambda}_{B, H}$ exists for all values of $k$ and $r$ if $x_{(k)}<T$.

The hyper parameter $A$ is assumed to equal $r / \sum_{i=1}^{r} x_{(i)}$ if $x_{(k)}<T$ and $k / \sum_{i=1}^{k} x_{(i)}$ if $x_{(k)}>T$. The hyper parameter $B$ is assumed to equal $x_{(1)}$ in both cases.

Ganguly et. al. [3] showed that based on the Type-II hybrid censored sample the MLE of $\theta$ does not exist when $k=1$ and $r=0$. So, conditional MLEs, conditioning on the event $r \geq 1$ when $k=1$ were proposed by Ganguly et. al. [3] as follows:

$$
\hat{\lambda}_{M L E, H}=x_{(1)} \text { and } \hat{\theta}_{M L E, H}=\frac{r}{\left[\sum_{i=2}^{r} x_{(i)}-(n-1) x_{(1)}+(n-r) T\right]}
$$

But when $k \geq 2$ the MLEs of the scale and location parameters exist for all values of $r$ and given by:

$$
\hat{\lambda}_{M L E, H}=x_{(1)} \text { and } \hat{\theta}_{M L E, H}= \begin{cases}\frac{r}{\left[\sum_{i=2}^{r} x_{(i)}-(n-1) x_{(1)}+(n-r) T\right]} & \text { if } x_{(k)}<T \\ \frac{k}{\left[\sum_{i=2}^{k-1} x_{(i)}-(n-1) x_{(1)}+(n-k+1) x_{(k)}\right]} & \text { if } x_{(k)}>T\end{cases}
$$




\section{Credible Intervals}

\subsection{Based on Complete Sample}

Based on the complete sample $x_{1}, x_{2}, \ldots, x_{n}$ and by using the posterior density function of $\theta$, the equaltailed $(1-\alpha) 100 \%$ credible interval for $\theta$ denoted by $\left(\theta_{L}, \theta_{U}\right)$ can be obtained numerically by solving the following nonlinear equations:

$$
\begin{gathered}
\frac{1}{C \Gamma(n)}\left\{D^{-n}\left[\Gamma(n, 0)-\Gamma\left(n, D \theta_{L}\right)\right]-E^{-n}\left[\Gamma(n, 0)-\Gamma\left(n, E \theta_{L}\right)\right]\right\}=\alpha / 2 \\
\frac{1}{C \Gamma(n)}\left\{\Gamma(n)\left\{D^{-n}-E^{-n}+\theta_{U}\left[\left(D \theta_{U}\right)^{-n}-\left(E \theta_{U}\right)^{-n}\right]\right\}+D^{-n} \Gamma\left(n, D \theta_{U}\right)-E^{-n} \Gamma\left(n, E \theta_{U}\right)\right\}=\alpha / 2
\end{gathered}
$$

where $\Gamma(a, b)=\int_{b}^{\infty} x^{a-1} e^{-x} d x$, the incomplete gamma function.

Similarly, by using the posterior density function of $\lambda$, the equal-tailed $(1-\alpha) 100 \%$ credible interval for $\lambda$ can be easily derived as:

$$
\left(-\frac{1}{n}\left[\left(\frac{C \alpha}{2}+F^{-n}\right)^{-1 / n}-F\right],-\frac{1}{n}\left[\left(\frac{2}{C \alpha}-(F-n B)^{-n}\right)^{-1 / n}-F\right]\right)
$$

in which $F=\sum_{i=1}^{n} x_{i}+A$.

\subsection{Based on Type-II Hybrid Censored Sample}

Based on a Type-II hybrid censored sample and by using the posterior density function of $\theta$, the equal-tailed $(1-\alpha) 100 \%$ credible interval for $\theta$ denoted by $\left(\theta_{L, H}, \theta_{U, H}\right)$ can be obtained numerically by solving the following nonlinear equations:

- If $x_{(k)}<T$ :

$$
\begin{aligned}
& \frac{1}{C_{1} \Gamma(r)}\left\{D_{1}^{-r}\left[\Gamma(r, 0)-\Gamma\left(r, D_{1} \theta_{L, H}\right)\right]-E_{1}^{-r}\left[\Gamma(r, 0)-\Gamma\left(r, E_{1} \theta_{L, H}\right)\right]\right\}=\alpha / 2 \\
& \frac{1}{C_{1} \Gamma(r)}\left\{\begin{array}{l}
\Gamma(r)\left\{D_{1}^{-r}-E_{1}^{-r}+\theta_{U, H}\left[\left(E_{1} \theta_{U, H}\right)^{-r}-\left(E_{1} \theta_{U, H}\right)^{-r}\right]\right\}+ \\
D_{1}^{-r} \Gamma\left(r, D_{1} \theta_{U, H}\right)-E_{1}^{-r} \Gamma\left(r, E_{1} \theta_{U, H}\right)
\end{array}\right\}=\alpha / 2
\end{aligned}
$$

- If $x_{(k)}>T$ : 


$$
\begin{gathered}
\frac{1}{C_{2} \Gamma(k)}\left\{D_{2}^{-k}\left[\Gamma(k, 0)-\Gamma\left(k, D_{2} \theta_{L, H}\right)\right]-E_{2}^{-k}\left[\Gamma(k, 0)-\Gamma\left(k, E_{2} \theta_{L, H}\right)\right]\right\}=\alpha / 2 \\
\frac{1}{C_{2} \Gamma(k)}\left\{\begin{array}{l}
\Gamma(k)\left\{D_{2}^{-k}-E_{2}^{-k}+\theta_{U, H}\left[\left(E_{2} \theta_{U, H}\right)^{-k}-\left(E_{2} \theta_{U, H}\right)^{-k}\right]\right\}+ \\
D_{2}^{-k} \Gamma\left(k, D_{2} \theta_{U, H}\right)-E_{2}^{-k} \Gamma\left(k, E_{2} \theta_{U, H}\right)
\end{array}\right\}=\alpha / 2
\end{gathered}
$$

Similarly, by using the posterior density function of $\lambda$, the equal-tailed $(1-\alpha) 100 \%$ credible interval for $\lambda$ can be easily derived as:

- If $x_{(k)}<T$ :

$$
\left(-\frac{1}{n}\left[\left(\frac{C_{1} \alpha}{2}+F_{1}^{-r}\right)^{-1 / r}-F_{1}\right],-\frac{1}{n}\left[\left(\frac{2}{C_{1} \alpha}-\left[F_{1}-n B\right]^{-r}\right)^{-1 / r}-F_{1}\right]\right)
$$

in which $F_{1}=A+\sum_{i=1}^{n} x_{i} \delta_{i}+T(n-r)$.

- If $x_{(k)}>T$ :

$$
\left(-\frac{1}{n}\left[\left(\frac{C_{2} \alpha}{2}+F_{2}{ }^{-k}\right)^{-1 / k}-F_{2}\right],-\frac{1}{n}\left[\left(\frac{2}{C_{2} \alpha}-\left[F_{2}-n B\right]^{-k}\right)^{-1 / k}-F_{2}\right]\right)
$$

in which $F_{2}=A+\sum_{i=1}^{n} x_{i} \delta_{i}+x_{(k)}(n-k)$.

\section{Example: Real Lifetime Data}

In this section we analyze a data set from Grubbs [6] to illustrate our methodology; the data summarizes the mileages at which nineteen military carriers failed. These were:

$162,200,271,302,393,508,539,629,706,777,884,1101,1182,1463,1603,1984,2355,2880$

The parameters $\theta$ and $\lambda$ are estimated by the MLE and the proposed Bayes estimates based on the complete sample and based on a Type-II hybrid censored sample with arbitrary $r=12$ and $T=800$ at which $k=10$.

Based on the complete sample it can be easily observed that

$$
\hat{\theta}_{M L E, C}=0.011973, \hat{\lambda}_{M L E, C}=x_{(1)}=162 \text {. }
$$


$\hat{\theta}_{\text {Bayes, } C}=0.0120424, \quad \hat{\lambda}_{\text {Bayes }, C}=121.7$

Based on the Type-II hybrid censored sample it can be easily observed that

$$
\begin{aligned}
& \hat{\theta}_{M L E, H}=0.00115864, \hat{\lambda}_{M L E, H}=162 . \\
& \hat{\theta}_{\text {Bayes }, H}=0.00117087, \quad \hat{\lambda}_{M L E, H}=120.589 .
\end{aligned}
$$

It has been observed that the MLEs and the Bayes estimates are very close to each other based on both the complete and the Type-II hybrid censored sample. One of the natural questions is whether the two-parameter exponential distribution fits this data set or not. There are several methods to test goodness of fit of a particular distribution to a given data set. The most suitable one in our case in the Kolmogorov-Smirnov (KS) test as the available sample size is small.

The KS test statistics have been computed based on the MLEs and the proposed Bayes estimates, they respectively equal: 0.0607852 and 0.0561898 (based on the complete sample), and 0.0621901 and 0.0488786 (based on the Type-II hybrid censored sample). The critical value of the KS test equals 0.27851 at $95 \%$ confidence level and at $n=18$, and it equals 0.33815 at $r=12$. Therefore, based on the KS test statistics and the critical values we could say that the MLE and the proposed Bayes estimates work quite well but the Bayes estimates performs slightly better than the MLE because it gives smaller values of KS test statistics based on both complete and censored samples.

On another hand, the $95 \%$ Credible intervals for the parameters $\theta$ and $\lambda$ are respectively $(0.000792871,0.268425)$ and $(44.9362,997.211)$ based on the complete sample, and $(0.000684133$, $0.00176701)$ and $(41.999,707.105)$ based on the Hybrid censored sample. These credible intervals seem to give reasonable estimates for the unknown parameters.

\section{Simulation Studies}

In this section, the performance of the proposed Bayes estimators of $\theta$ and $\lambda$ is investigated through simulation studies based on complete and Type-II hybrid censored samples. The simulation studies are carried out for different values of the combination $(\theta, \lambda, n, k, T)$. In these studies, we have generated 1000 random samples of size $n(=5$ and 30) from a two parameter exponential distribution, $E(\theta, \lambda)$, with various values for the parameters $(\theta, \lambda)$. For the purpose of comparison, the average values of the Bayes estimators $\hat{\lambda}_{B, H}$ and $\hat{\theta}_{B, H}$, and the MLEs $\hat{\lambda}_{M L E, H}$ and $\hat{\theta}_{M L E, H}$, along with their MSE, in parentheses, are reported in Table 1 and Table 2 based on various Type-II hybrid censoring schemes. The true location and scale parameters are assumed to equal $(\theta, \lambda)=(0.5,1),(3.5,1),(6.5,1)$, $(0.5,10),(3.5,10)$ and $(6.5,10)$. For simplicity, the hyper parameters $A$ and $B$ are assumed to equal the inverse of the mean of the available sample and the minimum observation respectively. Estimators with the smallest MSE values in the most cases are preferred. 
Table 1: MLE and BE along with their MSE based on various Type-II Hybrid censoring Schemes when $\lambda=1$ and $\theta=0.5,3.5$ and 6.5 .

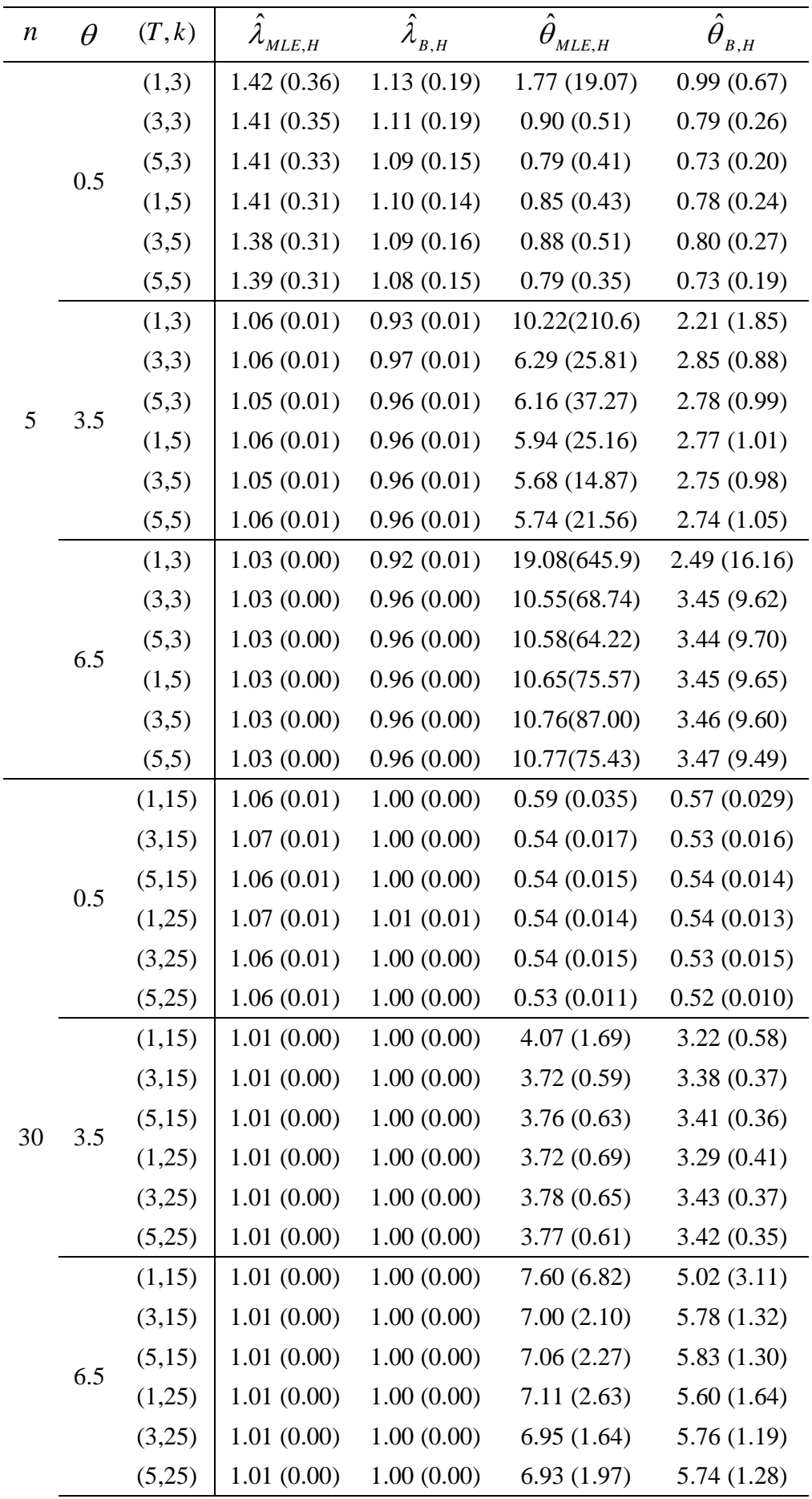


Table 2: MLE and BE along with their MSE based on various Type-II Hybrid censoring Schemes when $\lambda=10$ and $\theta=0.5,3.5$ and 6.5 .

\begin{tabular}{|c|c|c|c|c|c|c|}
\hline$n$ & $\theta$ & $(T, k)$ & $\hat{\lambda}_{M L E, H}$ & $\hat{\lambda}_{B, H}$ & $\hat{\theta}_{M L E, H}$ & $\hat{\theta}_{B, H}$ \\
\hline \multirow{18}{*}{5} & \multirow{6}{*}{0.5} & $(10,3)$ & $10.41(0.33)$ & $10.00(0.23)$ & $1.49(6.17)$ & $1.32(3.17)$ \\
\hline & & $(12,3)$ & $10.42(0.35)$ & $10.00(0.24)$ & $0.92(0.53)$ & $0.89(0.47)$ \\
\hline & & $(14,3)$ & $10.41(0.33)$ & $9.96(0.25)$ & $0.84(0.46)$ & $0.82(0.41)$ \\
\hline & & $(10,5)$ & $10.43(0.37)$ & $10.02(0.22)$ & $0.80(0.34)$ & $0.78(0.31)$ \\
\hline & & $(12,5)$ & $10.40(0.32)$ & $10.00(0.18)$ & $0.88(0.62)$ & $0.86(0.54)$ \\
\hline & & $(14,5)$ & $10.39(0.30)$ & $9.99(0.18)$ & $0.81(0.38)$ & $0.80(0.34)$ \\
\hline & \multirow{6}{*}{3.5} & $(10,3)$ & $10.05(0.01)$ & $9.99(0.00)$ & $9.74(132.3)$ & $6.46(24.39)$ \\
\hline & & $(12,3)$ & $10.06(0.01)$ & $10.00(0.00)$ & $5.82(17.79)$ & $5.07(8.82)$ \\
\hline & & $(14,3)$ & $10.05(0.01)$ & $9.99(0.00)$ & $5.90(30.08)$ & $5.04(10.91)$ \\
\hline & & $(10,5)$ & $10.06(0.01)$ & $9.99(0.00)$ & $5.97(25.53)$ & $5.13(10.67)$ \\
\hline & & $(12,5)$ & $10.06(0.01)$ & $10.00(0.00)$ & $5.93(20.08)$ & $5.13(9.66)$ \\
\hline & & $(14,5)$ & $10.06(0.01)$ & $10.00(0.00)$ & $5.89(19.68)$ & $5.11(9.72)$ \\
\hline & \multirow{6}{*}{6.5} & $(10,3)$ & $10.03(0.00)$ & $9.99(0.00)$ & $19.39(3526)$ & $9.07(29.43)$ \\
\hline & & $(12,3)$ & $10.03(0.00)$ & $10.00(0.00)$ & $10.49(63.72)$ & $8.26(18.15)$ \\
\hline & & $(14,3)$ & $10.03(0.00)$ & $9.99(0.00)$ & $11.48(103.9)$ & $8.77(23.94)$ \\
\hline & & $(10,5)$ & $10.03(0.00)$ & $9.99(0.00)$ & $10.72(54.23)$ & $8.49(16.90)$ \\
\hline & & $(12,5)$ & $10.03(0.00)$ & $9.99(0.00)$ & $10.87(72.46)$ & $8.51(18.83)$ \\
\hline & & $(14,5)$ & $10.03(0.00)$ & $9.99(0.00)$ & $10.57(59.66)$ & 8.35 (17.38) \\
\hline \multirow{18}{*}{30} & \multirow{6}{*}{0.5} & $(10,15)$ & $10.06(0.01)$ & $10.00(0.00)$ & $0.58(0.03)$ & $0.58(0.03)$ \\
\hline & & $(12,15)$ & $10.07(0.01)$ & $10.00(0.01)$ & $0.54(0.02)$ & $0.54(0.02)$ \\
\hline & & $(14,15)$ & $10.06(0.01)$ & $9.99(0.00)$ & $0.54(0.01)$ & $0.54(0.01)$ \\
\hline & & $(10,25)$ & $10.07(0.01)$ & $10.00(0.00)$ & $0.54(0.02)$ & $0.54(0.02)$ \\
\hline & & $(12,25)$ & $10.06(0.01)$ & $10.00(0.00)$ & $0.55(0.02)$ & $0.55(0.02)$ \\
\hline & & $(14,25)$ & $10.06(0.01)$ & $10.00(0.00)$ & $0.54(0.01)$ & $0.54(0.01)$ \\
\hline & \multirow{6}{*}{3.5} & $(10,15)$ & $10.01(0.00)$ & $10.00(0.00)$ & $4.11(1.92)$ & $3.99(1.60)$ \\
\hline & & $(12,15)$ & $10.01(0.00)$ & $10.00(0.00)$ & $3.69(0.48)$ & $3.65(0.44)$ \\
\hline & & $(14,15)$ & $10.01(0.00)$ & $10.00(0.00)$ & $3.75(0.51)$ & $3.71(0.47)$ \\
\hline & & $(10,25)$ & $10.01(0.00)$ & $10.00(0.00)$ & $3.85(0.81)$ & $3.79(0.73)$ \\
\hline & & $(12,25)$ & $10.01(0.00)$ & $10.00(0.00)$ & $3.74(0.64)$ & $3.69(0.59)$ \\
\hline & & $(14,25)$ & $10.01(0.00)$ & $10.00(0.00)$ & $3.73(0.55)$ & $3.68(0.50)$ \\
\hline & \multirow{6}{*}{6.5} & $(10,15)$ & $10.01(0.00)$ & $10.00(0.00)$ & $7.52(5.81)$ & 7.13 (4.18) \\
\hline & & $(12,15)$ & $10.01(0.00)$ & $10.00(0.00)$ & $7.02(2.12)$ & $6.86(1.81)$ \\
\hline & & $(14,15)$ & $10.01(0.00)$ & $10.00(0.00)$ & $6.90(1.90)$ & $6.74(1.64)$ \\
\hline & & $(10,25)$ & $10.01(0.00)$ & $10.00(0.00)$ & $7.08(2.55)$ & $6.87(2.10)$ \\
\hline & & $(12,25)$ & $10.01(0.00)$ & $10.00(0.00)$ & $6.91(2.18)$ & $6.76(1.88)$ \\
\hline & & $(14,25)$ & $10.01(0.00)$ & $10.00(0.00)$ & $6.90(1.90)$ & $6.73(1.64)$ \\
\hline
\end{tabular}

Table 1 and Table 2 show that Type-II hybrid censoring is an efficient censoring scheme to estimate the location and scale parameters of a two-parameter exponential distribution. 
It can be seen from Table 1 and Table 2 that the proposed Bayes estimates $\hat{\lambda}_{B, H}$ and $\hat{\theta}_{B, H}$ give excellent results, and dominate in terms of MSE the MLEs in all cases. It can be also concluded that when $n$ is large, the MLE and the proposed Bayes estimators give quite similar results, with very small values of MSE. When $n$ is small, the proposed Bayes estimators $\hat{\lambda}_{B, H}$ and $\hat{\theta}_{B, H}$ give excellent results for estimation the location and scale parameters, and perform in terms of MSE better than the MLEs. It is noticed also from Tables 2 and 3 that, the MLE of $\theta$ gives bad results in some cases such as, when the true $\theta=3.5$ and based on the first censoring scheme.

It can be also seen from Table 1 and Table 2 that the proposed Bayes estimates $\hat{\lambda}_{B, H}$ and $\hat{\theta}_{B, H}$ are not as sensitive to the changes in the parameters $n, T$ and $k$ as the MLEs $\hat{\lambda}_{M L E, H}$ and $\hat{\theta}_{M L E, H}$ are.

\section{Conclusions}

In this paper, we have considered the Type-II hybrid censoring scheme when lifetimes have a twoparameter exponential distribution from Bayes point of view. Prior exponential and uniform probability distributions were assumed for the scale and location parameters respectively. MLEs, Bayes point estimates and credible intervals for $\theta$ and $\lambda$ were proposed based on complete and Type-II hybrid censored samples under the squared error loss. The Bayes estimates for both parameters have been derived in closed forms. Credible intervals of the location parameter were derived also in closed forms based on the complete and the Type-II censored samples, but it could not be derived in closed form for the scale parameter. It was shown from real data set and from simulation studies that the proposed Bayes estimates $\hat{\lambda}_{B, H}$ and $\hat{\theta}_{B, H}$ gave excellent results and dominate, in terms of MSE, the maximum likelihood estimates in all cases. Moreover, it was observed in some cases that the MLE of $\theta$ gives very large MSE values. So, it was recommended to use the proposed Bayes estimates $\hat{\lambda}_{B, H}$ and $\hat{\theta}_{B, H}$ to estimate the parameters of the two-parameter exponential distribution based on complete and Type-II hybrid censored samples.

\section{References}

[1] A. Childs, B. Chandrasekar, N. Balakrishnan and D. Kundu, Exact likelihood inference based on Type-I and Type-II hybrid censored samples from the exponential distribution, Annals of the Institute of Statistical Mathematics 55 (2003) 319-330.

[2] A. Childs, N. Balakrishnan, and B. Chandrasekar, Exact distribution of the MLEs of the parameters and of the quantiles of two-parameter exponential distribution under hybrid censoring, Statistics 46 (2012) 441458.

[3] A. Ganguly, S. Mitra, D. Samanta and D. Kundu, Exact inference for the two-parameter exponential distribution under Type II hybrid censoring, J. Statist. Plann. Inference 142(3) (2012) 613-625.

[4] B. Epstein, Truncated life-test in exponential case, Annals of Mathematical Statistics 25 (1954) 555-564. 
[5] B. Prasad and R.S. Singh, Estimation of prior distribution and empirical Bayes estimation in a nonexponential family, J. Statist. Plann. Inference 24 (1990) 81-86.

[6] F.E. Grubbs, "Approximate Fiducial Bounds on Reliability for the Two Parameter Negative Exponential Distribution," Technometrics 13 (1971) 873-876.

[7] H. Bayoud, Bayesian Analysis of Type I Censored Data from Two-Parameter Exponential Distribution, Lecture Notes in Engineering and Computer Science 2197 (2012) 291-296.

[8] MIL-STD-781-C. Reliability Design Qualification and Production Acceptance Tests: Exponential Distribution. (U.S. Government Printing Office-Washington, D.C, 1977).

[9] N. Balakrishnan and A.P. Basu. The Exponential Distribution: Theory, Methods and Applications. (Gordon and Beach Publishers-Netherlands, 1995)

[10] N. Draper and I. Guttman, Bayesian analysis of hybrid life tests with exponential failure times, Annals of the Institute of Statistical Mathematics 39 (1987) 219-225.

[11] R.S. Singh and B. Prasad, Uniformly strongly consistent prior distribution and empirical Bayes estimators with asymptotic optimality and rates in a non-exponential family, Sankhya A 51 (1989) 334-342. 